\title{
Corporate survival in Industry 4.0 era: the enabling role of digital twin technologies
}

\author{
Natalia Serbulova ${ }^{1 *}$ \\ ${ }^{1}$ Don State Technical University, 1 Gagarin sq., Rostov-on-Don, 344003, Russia
}

\begin{abstract}
In Industry 4.0 era high-tech companies have digitally transformed production and have taken the lead globally. Among the many advanced technologies, the digital twin is the unifier of many cross-cutting digital technologies, acting as a driver of digital transformation, enabling breakthroughs and allowing companies to move to new levels of sustainability and industrial leadership in global markets. The paper has sought to identify generalized characteristics of the digital twins. The results can be used to classify what is a digital twin. After providing a digital twin definition and characterization, the process of practical implementation of digital twins in different industries has been examined. The key drivers and limiting factors of digital twin implementation strategies are also discussed to inspire future studies. The research was based on the digital twins' impact analysis on various industries using leading industry data sources, analytical reports, international indexes and indicators.
\end{abstract}

\section{Introduction}

In 2018, global research and advisory company Gartner has listed digital twins (DT) among the top 10 strategic technology trends [1]. In 2019, Russia adopted a roadmap for the development of «end-to-end» digital technology «New Manufacturing Technologies». The program notes that, among many advanced technologies, the digital twin is the integrator of almost all «end-to-end» digital technologies and sub-technologies, acting as a driver, providing breakthroughs and enabling companies to move to a new level of sustainable development on their way to industrial leadership in global markets.

In business terms, digital twin technology shows great promise because the customer needs DT not only to operate a modern smart product, but also to maintain it regularly, receive updates and continuously adapt to new requirements and conditions. Many companies have already incorporated or are in the process of incorporating data, information, and analytics capabilities as part of their service offerings [2].

The customers already need a physical product in a «digital bundle», they are already prepared to pay for both a physical object and a virtual one. In the near future, in highly competitive markets, it will only be possible to sell a product if you have both the product and its digital counterpart at the same time.

\footnotetext{
*Corresponding author: nserbulova@mail.ru
} 
The concept of digital twins as a virtual representation of a physical object has been used for over 30 years. In recent years, however, there has been a quantum leap in the development and application of this technology due to the total digitalization of industrial enterprises. Security requirements are increasing, and data volumes are growing every day. Organizations along various industries tend to inculcate digital twin in their business.

The existing literature highlights many of the potential and perceived benefits of the Digital Twin concept, including reducing costs and risk, improving efficiency and service offerings, security, reliability and resilience, and supporting decision-making process [312].

The increased complexity of products has required a change in product strategy. The challenges are related to the increased part counts, and the fact that electronics, software and embedded systems have become an integral part of any high-tech product, and the integration of these parts becomes a separate task.

The reliability and safety of a multicomponent product requires a dramatic multiplication of tests, which are quicker and cheaper to do in virtual space at the design stage than at a later stage in material. Creating a competitive product requires optimizing tens of thousands of parameters and using digital twin technology.

Despite the great interest in DT, community of professionals has not yet agreed-upon DT definition and regulation. Professionals are still debating what a digital twin is, what types of digital twins exist, in which industry solutions the term is applicable, etc. The answers to these questions require a sufficiently broad analysis of the DT implemented options on various machine types, mechanisms and constructions, in different industries and countries.

There is a need for examples of practical implementations of Digital Twins that consider the implementation strategies and decision support to achieve targeted outcomes with measurable benefits.

This paper evaluates the status of Digital Twins as part of a family of digitalization efforts intended to enhance existing processes and support new services. The contributions of the paper are as follows. First, existing Digital Twin definitions are reviewed and a consolidated definition and description of the key characteristics of the Digital Twin is provided. The approach and considerations for the implementation of Digital Twins for practical applications are discussed. The implementation approach and related considerations are then demonstrated through the industry case studies. Finally, current growth drivers and constraints in the Digital Twin market are discussed.

\section{Digital twin definition and characterization}

The term digital twin has had different emphases at different times. It originated within the engineering paradigm, in relation to industrial products, where there was a clear link between the digital twin and the real object at all stages of the product life cycle. This concept is still the mainstream today. However, over time, references to the digital twin of processes began to appear, where a clear link to physical and technological processes cannot always be traced. There appeared publications, where the digital twin appeared not only as the products of industrial production, but also as the modeling of natural objects the phrases like «digital twin of the reservoir» or «human digital twin» appeared. The term has been interpreted more and more broadly within different applications in different fields, and specialists have adapted it to their areas of application. Engineers were followed by oil companies, retailers, doctors, all talking about creating digital twins.

A digital twin is a virtual prototype of a real object, group of objects or processes. It is a complex software product that is created from a variety of data. The digital twin is not limited to collecting data from the product engineering and production stages - it continues 
to collect and analyze data throughout the lifecycle of the real object, including through the use of numerous IoT sensors. The technology makes it possible to simulate a wide variety of situations that may arise in production. In this way, the digital twin enables the most appropriate process scenarios to be selected in order to avoid disruptions and force majeure circumstances.

It can be said that IoT technologies and digital twins are complementary. The dramatic growth in the number of IoT devices and their cost reduction are driving forces in the use of digital twins. Improvements in smart IoT sensors enable digital twins to apply increasingly compact solutions. In addition, the digital twin enables a number of challenges that arise within IoT projects. As more and more interconnected IoT things are introduced, management of data, systems and devices is becoming increasingly complex. The digital twin allows the IoT system to be structured and organized. Complex product DT is usually a collection of physical subsystems' twins. Instead of communicating with each subsystem separately, the physical object (e.g. a machine tool) sends all data to its digital twin. The digital twin aggregates and processes this data, and those subsystems that require information are connected directly to the digital twin where they can access the data.

A digital twin of the plant should be able to simulate situations based on factors ranging from equipment location, worker movement and repair operations to the response of the instruments to changes in sunlight or some kind of emergency situation. These are just examples of tests, simulations and impacts. It all depends on the business objectives and the type of product or object.

Sensors are built into the physical object which collect real-time data about the state of that object, the data is sent to a digital twin; a digital model is refined on the basis of the received data. The model takes into account all changes occurring to the physical object, accumulates information about its behavior and as it refines it can more adequately describe and predict the behavior of the physical object. Based on the updated model, recommendations for optimizing the operation and maintenance of the real object can be generated. For example, the model can predict the possibility of failure of a particular component, recommend timing of preventive maintenance, maintenance inspections, filter changes, etc.

Numerical modeling techniques of the physical processes in the materials of an object are used to construct a complex twin model. This helps to predict the product response to operating loads, e.g. by using the Finite Element Analysis (FEA) method. With this method, it is possible to simulate the behaviour of complex systems by dividing them into sets of elements (cells) small enough to consider their properties as homogeneous. The method is widely used for solving problems in solid mechanics, heat transfer, fluid dynamics and electrodynamics. CAD-models (computer aided design/drafting) are also used to provide information on appearance and structure of objects, materials, processes, dimensions and other parameters. FMEA (Failure Mode and Effects Analysis) models based on reliability analysis are also used.

Between 2010 and 2014, the concept of digital twins evolved with close attention from the aerospace industry, and the term was applied to industrial product design, predominantly for transport. What were the distinctive features of the new concept at this stage? Combining different sources, the following list can be cited:

- DT is a digital copy of a specific physical object that reflects the structure, performance, technical condition and nature of the physical object's mission, including parameters such as kilometer performance, emerged malfunctions, and maintenance and repair history of the actual product (the physical twin);

- DT is based on MultiPhysics, a mathematical modeling of different physical processes that determine the properties and behavior of an object; 
- DT is associated with specific conditions in which the real object operates. It is a model that accumulates information about a real product as it operates under specific conditions. Two structurally identical products will have different digital twins if they operate under different conditions;

- DT allows developers to monitor the virtual object's performance in order to better understand how to optimize the physical object's performance;

- DT helps to understand how the physical twin (the real object) performs in the real world, and can give a prediction of how this work will be performed in the event of timely maintenance in the future;

- DT allows collecting data about a physical object and, using predictive analytics tools, making predictions about the condition of that object, determining when preventive maintenance should be scheduled;

- DT allows correcting malfunctions of remote equipment and remote maintenance;

- DT provides data that cannot be obtained directly from a physical object. This can be used as a tool to troubleshoot existing products and optimize the performance of subsequent generations.

Some definitions refer only to the DT's virtual part, which is defined as a virtual model consisting of two parts - the Digital master and the Digital shadow. The Digital master contains sufficient information to produce a product with customer-specified properties, including an analysis of the physical properties of that product. The Digital shadow, which the physical object «casts into virtual space», is a collection of sensor data and a model that allows predicting object properties within certain limits. The combination of the Digital master and the Digital shadow defines the concept of the virtual part of the digital twin. As a process, they began not only to consider the process of assembling a product in discrete productions, but also began to talk about digital twins of process production and logistics. There are such concepts as, for example: «Digital oilfield twin», «Digital twin of retail store» [13].

The process of forming the concept of the digital twin is ongoing. On the one hand, the digital twin has become a popular term; on the other hand, it has not outlived the features of the so-called pre-terminus. A «pre-terminus» is used as a term for new concepts, but it does not meet the basic requirements for a term, such as unambiguity, precision, lack of synonyms, relevance to a scientific discipline, systematic approach, definiteness (i.e. having an unambiguous definition).

CAD models that carry information about the geometry of the product (appearance and structure of objects), information about materials, processes, dimensions, etc.; they are the means for modeling physical processes in the materials of the object, for example, based on Finite Element Analysis (FEA) method. These are also models based on Failure Mode and Effects Analysis (FMEA), based on system reliability prediction, which can combine mathematical models of failure with a statistical database of failure modes, as well as data on the maintenance and product operation history [14]. By combining data from various sources of information, the digital twin can predict the technical state of a physical object. The DT can also be used to predict the system's response to critical safety events. The DT can generate control actions that can mitigate damage or degradation of systems by activating self-healing mechanisms or recommending changes to the operational mission profile (e.g. by selecting a mode with less stress on the problem area, thereby increasing both lifetime and the probability of mission success). A digital twin links the virtual and physical environments. The physical environment (real object, built-in and external sensors) constantly transmits operation and maintenance data to update the virtual model in the digital twin. In this way, the DT becomes an accurate real-time representation of the physical system, whenever it changes. 
The digital twin uses real-time measurement data. This information is supplemented by metadata, properties and documents, such as reports or operating procedures, generated during all stages of the object lifecycle. Different types of information and technologies can be used at different stages in the DT.

Table 1. Information and technologies that can be used in the DT at different stages.

\begin{tabular}{|c|c|c|c|c|}
\hline & Planning & Creating & Operation & Maintenance \\
\hline $\begin{array}{c}\text { Product } \\
\text { information at } \\
\text { different stages } \\
\text { of the life cycle }\end{array}$ & PLM & PLM & $\begin{array}{l}\text { Operational } \\
\text { Instructions }\end{array}$ & $\begin{array}{l}\text { Service } \\
\text { Delivery } \\
\text { Records }\end{array}$ \\
\hline $3 \mathrm{D}$ view & $\begin{array}{l}\text { Design } \\
\text { drawings }\end{array}$ & $\begin{array}{c}\text { Standard } \\
\text { operating } \\
\text { procedures }\end{array}$ & $\begin{array}{c}\text { Object } \\
\text { visualization }\end{array}$ & $\begin{array}{c}\text { Service } \\
\text { instructions, } \\
\text { augmented } \\
\text { reality (AR) }\end{array}$ \\
\hline Simulation & $\begin{array}{l}\text { Behavior } \\
\text { prediction }\end{array}$ & $\begin{array}{c}\text { Virtual } \\
\text { commissioning }\end{array}$ & $\begin{array}{c}\text { Performance } \\
\text { monitoring }\end{array}$ & $\begin{array}{c}\text { Diagnostic } \\
\text { operation }\end{array}$ \\
\hline $\begin{array}{l}\text { Numerical } \\
\text { Modelling }\end{array}$ & $\begin{array}{l}\text { Design } \\
\text { modelling }\end{array}$ & $\begin{array}{c}\text { Hardware in the } \\
\text { loop (HIL) } \\
\text { simulation }\end{array}$ & $\begin{array}{l}\text { What-if } \\
\text { scenarios }\end{array}$ & Forecasting \\
\hline Model Data & $\begin{array}{c}\text { Engineering } \\
\text { data }\end{array}$ & Production data & $\begin{array}{c}\text { Operational } \\
\text { data }\end{array}$ & Service data \\
\hline $\begin{array}{c}\text { Related } \\
\text { Analytics }\end{array}$ & - & - & KPIs & KPIs \\
\hline Visualization & $\begin{array}{l}\text { Development } \\
\text { tools }\end{array}$ & $\begin{array}{l}\text { Development } \\
\text { tools }\end{array}$ & $\begin{array}{l}\text { Visual image of } \\
\text { the operating } \\
\text { condition }\end{array}$ & $\begin{array}{c}\text { Visual image of } \\
\text { the technical } \\
\text { diagnostics }\end{array}$ \\
\hline
\end{tabular}

\section{Digital Twin across different industries}

Digital transformation is viewed as an enabler of more innovative, optimized, and effective products and processes and is a key component of Industry 4.0 [15].

Until now, the concept of a digital twin has been considered mainly in relation to industrial products, but now the interpretation of the term is expanding. Indeed, the term is used in different industries and each industry has its own specifics. Digital twin technology can be used in modeling human behavior as a recipient of certain services. Similar projects are being implemented in retail, financial services, and insurance, where personalized profiles of individuals can be created and the appropriateness of financial decisions can be analyzed.

Information and communication technologies (ICT) can be leveraged to design and implement the next generation of data, models, and decision support tools for agricultural production systems [16]. Agricultural DT range from individual plants and animals to twins of land parcels, farms, or regions [17]. Dutch startup Connecterra has created a digital twin to remotely monitor the health of cattle. The solution is based on a model that uses artificial intelligence to process data obtained from IoT sensors and suggests a farmer how to optimize the care of the animal at different stages of its lifecycle. The BeeZon application, developed by Greek specialists, continuously monitors apiaries in real time. BeeZon helps beekeepers remotely monitor apiaries and life of bee colonies by monitoring the temperature, humidity and acoustic background in each hive, to make correct and quick decisions when any problems arise.

The emergence of digital twin technologies in architectural and structural design has made a huge difference. Using DT has made it possible to build a «smart house» in its full 
sense, including not only digital design, real-time operation, but also intelligent real estate management as an asset.

Many countries and governments consider smart cities a solution to global warming, population growth, and resource depletion. Digital twins, along with the IoT, 5G, blockchain, collaborative computing, simulation, and AI technologies, offer great potential in the transformation of the current urban governance paradigm toward sustainable smart cities $[18,19]$.

The concept of DT has entered the medical field extensively, both in relation to the creation of digital models of individual organs (e.g. the digital twin heart), the 3D printing of organ analogs, and the increasing use of engineering approaches, technology and terminology in the planning and execution of surgical procedures. Personalized healthcare services based on analysis of patient data have much in common with DT technology used to create industrial products and processes. Advanced computer models of diseases can be used to improve diagnosis and treatment. The goal is to develop the models to DT of individual patients. DT may help to computationally identify and try the best medication, before actually treating a patient.

To become climate neutral by 2050, the European Union launched two ambitious programmes: «Green Deal» and «DigitalStrategy». As a key component of their successful implementation, climate scientists and computer scientists launched the «Destination Earth» initiative, which will start in mid-2021 and is expected to run for up to 10 years. During this period, a highly accurate digital model of the Earth is to be created, a digital twin of the Earth, to map climate development and extreme events as accurately as possible in space and time. The DT of the Earth is intended to be an information system that develops and tests scenarios that show more sustainable development and thus better inform policies. The digital twin will also be used for strategic planning of fresh water and food supplies or wind farms and solar plants.

Siemens and General Electric are considered to be the largest developers and market leaders in the DT market. These companies offer most of the components needed to create DT, including industrial IoT applications, advanced analytics and cloud platforms. Simcenter software (Siemens PLM Software) uses system simulation, 3D CAE and test to predict performance throughout the entire product lifecycle. For example, Simcenter Amesim simulates the complete aircraft flight cycle: preparation for takeoff, flight task execution and landing, taking into account the interaction of all aircraft systems.

General Electric (GE) has used DT to change their process of designing, manufacturing and maintaining their products, including engines, power generation, oil and gas and medical imaging equipment. GE has increased the reliability of some of their products by $93-99.49 \%$ in less than two years, reduced reactive maintenance by $40 \%$ in less than one year and avoided $\$ 11 \mathrm{~m}$ in lost productivity costs. For example, GE Transportation (now Wabtec) has created the Trip Optimizer, a digital twin of its Evolution locomotive. The DT simulates the most efficient speed and acceleration profile of the train based on the weather conditions, the topography of the train tracks and the train's scheduled arrival time. GE's Train Optimizer enables fuel savings of $3-17 \%$, or up to 32,000 gallons per engine annually.

In recent years, digital twin use has increased in property and construction, where the technology has demonstrated an impressive range and scale of benefits. A digital twin was developed for One Sydney Harbour, a Renzo Piano-designed residential development using the «approved for construction» design documents created by the design consultant teams, including models, 2D drawings, specifications and product tech data. The digital twin enabled unresolved design details and errors to be identified and resolved to a much finer level of detail than would have been achieved through a purely traditional approach and in a 
much shorter time frame. Digital twins have saved millions of dollars in construction costs and reduced construction time by months, while increasing the quality of the final product.

Digital twins are particularly powerful when used in conjunction with "generative design». Rather than using technology to help people design the building, the design is generated by automation software, in response to the design brief. Building options generated this way aren't simply drawings. They are a fully resolved solution from an engineering, performance and financial perspective, meaning that they are buildable, compliant and financially viable.

Founded in 2009, Chicago-based Cityzenith is the developer of Smart World Pro, a product that serves to apply DT technology to architecture at all stages of the product lifecycle, from design to demolition. Smart World Pro is used by architects, property managers and owners. In 2016, the product won the Realcomm Digie Award for the Best Tech Innovation: Commercial/Corporate Real Estate. Smart World Pro platform-based solution enables easy natural language search of project data, rapid ROI analysis based on $\mathrm{AI}$ and machine learning, and efficient customer engagement for deals.

Lendlease recently launched Podium, a digital platform for the real estate industry that harnesses the power of digital twins and generative design. Using the software, Lendlease has generated digital twins of buildings within several days, a process which would traditionally take a team of designers months to complete. Digital twins have therefore proven to make an important leap in productivity. Any improvement in productivity in the industry will have a major benefit for the world economy. Inefficiencies in property and construction are bad for the global economy, because they drive up the cost of construction, increasing the cost of infrastructure, property and houses. There is clearly a trade-off between increasing digitalization and environmental sustainability that needs to be carefully managed [20].

US electric car maker Tesla is another company that is investing in digital twin technology. The data collected from sensors allows it to monitor the operating condition of an electric vehicle, as well as detecting problems early on to avoid costly repairs. Tesla updates the software based on the sensor data and uploads updates to its products. This data-driven software development process allows for more efficient allocation of resources and significantly improved interaction with the electric vehicle user.

Boeing is moving towards model-based design by digitizing the entire design and development system, including supply chain information, data from the manufacturing system, and maintenance and support systems. As a result, the aircraft manufacturer has been able to improve the quality of the parts and systems it uses to produce commercial and military aircraft using digital twin technology by $40 \%$.

The Russian digital twin market is at an early stage of development, and there are no estimates of its size yet. However, there are a number of companies in Russia that can supply technology, components and individual products for building digital twins.

\section{Drivers and limiting factors of the DT market}

Analysts are unanimous in predicting rapid growth of the digital twin market. For example, according to Digital Twin Market Size, Share \& Trends Analysis Report By End-use (Automotive \& Transport, Retail \& Consumer Goods, Agriculture, Manufacturing, Energy \& Utilities), By Region, And Segment Forecasts, 2021 - 2028, published in April 2021, the global digital twin market size was valued at $\$ 5.04$ billion in 2020 . The market expected to expand at a compound annual growth rate of $42.7 \%$ from 2021 to 2028 according to Grand View Research report. The market experienced a slight slump in 2020 owing to the shutting down of manufacturing sites, production plants, and other factories in the first half of the year triggered by the spread of the COVID-19 pandemic [21]. 
According to Deloitte, the global market for digital twin technologies will grow to $\$ 16$ billion by 2023 . A report by Techsci Research, published in February 2018, gives roughly the same estimate.

This section highlights the drivers and limiting factors associated with developing and incorporating digital twins in practical applications in additional to some possible paths forward.

Speaking of drivers, first of all, the digital twin is one of the most important technologies that digital transformation projects rely on. It provides an opportunity to increase the competitive advantage of industrial products manufacturers, enables faster production, creates stronger and more reliable, less material-intensive structures (which is especially relevant given the rising cost of materials) and brings them to the market faster. This kind of manufacturing cannot be achieved without the use of digital twin technology. The increasing complexity of engineered objects requires a new design paradigm that overcomes the barriers between disintegrated design tools, enables integration among design teams as well as among multiple suppliers in complex supply chains. Digital twins reduce costs by shifting the "centre of gravity» to the design stage: the later the design changes, the more expensive the product is, and redesigning and multiple design refinements multiply both financial and time costs, thereby closing the window of opportunity for bringing new products to market.

Digital twins help to reduce costs at all other stages of the product lifecycle - during operation, maintenance, monitoring and disposal tasks. The market growth is strongly supported by examples of successful DT projects for unique products, showing the potential to extend the life of equipment, reduce repair costs, including examples of optimizing repairs in remote, difficult to access locations. Increasing demand for digital twin technology is growing with the development of related digital technologies: industrial IoT, cloud technologies, virtual and augmented reality applications, and additive manufacturing technologies. Reduced cost and increasing availability of low-power sensors and big data analytics applications, the availability of cloud computing power, and new opportunities to optimize the product for additive manufacturing technologies are driving the development of digital twin projects. In some companies, digital twin technologies are being developed as part of digitalization and transition to circular economy strategy, or as part of establishing an effective product safety program. Analytical and marketing companies conducted a number of surveys of professionals in order to assess the impact of the new technology. Among the main factors driving the adoption of DT are increased production quality, reduced cost of production and reduced unplanned downtime.

The analysis revealed a problem in the implementation of DT data interconnections. The implementation of digital twins requires decisions on how data will be collected, the frequency at which data will be collected, and how data will be exchanged between the physical and virtual spaces. Digital twin collects data primarily through sensor technologies. Additional data may also be obtained from expert opinions, pictures/video data, maintenance records, etc. The required technologies and processes to collect, process and use these data sources should be considered during implementation.

An important aspect of digital twin implementation is the frequency of data collection and exchange. There may be cases where sensor data is collected at a high frequency, but it is exchanged with the virtual representation only on a periodic basis in batch format due to data transmission limitations.

A final consideration relates to the technology and processes that must be implemented to support virtual-physical connection. Similar to the physical-to-virtual interconnection, this requires establishing the required data exchange processes and control systems to allow the DT to perform the specified actions. The implemented digital twin by design must be 
consistent with these processes, the points of information exchange and decision-making in the process must be identified.

The hindering factors preventing the development of the DT market are the relatively high cost of this type of projects, which requires multidisciplinary software and expertise in mathematical modeling of a wide range of physical processes that determine complex technological and production processes.

As the technology for creating digital twins improves, the threshold cost of economically viable implementation of digital twins will also decrease. There is also a shortage of specialists with the interdisciplinary knowledge required to create digital twin projects, and there is a lack of information and educational programmes on the subject.

The digital twin market is a young market, less than 10 years old, so there are still problems of consistency of interpretation of the term digital twin, and hence problems of correct estimation of the size of the digital twin market, its segmentation, assessment of the growth rate and, consequently, problems of assessing the attractiveness of the market to attract investment.

Promoting old technology under a new «label» discredits digital twin technology. To keep up with the new trends in the digital marketplace, some companies are marketing their products using the phrase «digital twin» in ways that are inappropriate. Such companies inadvertently create Digital Impostors - fragmented or incomplete digital twins. Such impostors compromise the value of digital twin technology by making solutions based on them expensive and poorly scalable, thus undermining the credibility of DT technology as an effective platform for digital transformation.

Another challenge to the adoption of digital twin technology is the so-called «vendor lock-in» fear. The fear of becoming dependent on the vendor of the digital twin is compounded by the fear of losing control over the information, of it falling outside the information security loop, and the reluctance of a community to share sensitive data for fear that the benefits of implementing a digital twin are less than the damage of losing control over a piece of information. This is not a new problem: on the one hand people need data integration, on the other hand people tend to set boundaries at the departmental, company and corporate level for security reasons, trying to control and limit the spread of data, thereby restraining the spread of digital twin technology.

Among the challenges is also a certain inertia in embracing the new culture of division of labor. Quite often engineering software vendors promote the idea that years of experience embedded in modern software allows engineers from developing countries to «stand on the shoulders" of specialists from developed countries (who have been creating this software for years) and even get ahead in design, avoiding the mistakes of the pioneers. Alas, practice does not abound in such examples, especially when it comes not to individual companies, but to an entire industry, or a number of industries, or a single country. A new technology cannot be absorbed instantly by its adherents, but takes time. And the old division of labor and the old culture of production are the main obstacles to its adoption.

\section{Conclusion}

Digital twins are based on a number of evolving technologies, so their development is directly dependent on the growth of the capabilities of these technologies. The growth of available computational resources for mathematic simulation plays a major role in the new capabilities of digital twins. Digital twins have become a really strong catalyst for the development of modern companies. They greatly simplify system maintenance, save resources, and minimize the risks of errors and failures, which prolongs the stable operation of the product. All this allows businesses to get the best possible return on investment, increase competitiveness and build customer loyalty. 
There are indicators that very soon consumers and product owners will be able to use a digital twin in everyday life, and the technology can be applied not only to improve the performance of a device, but also to improve the quality of human life. The new possibilities of artificial intelligence will allow creating digital twins, in which the role of a human being in making managerial decisions will be more and more minimised.

The process of merging digital twins will continue at different levels. The concept of national digital twins will be implemented, where individual DT will form networks of various kinds, collectively accumulating knowledge for different tasks. National solutions will be followed by Global.

Some of the challenges to digital twin implementation are technical in nature and can be resolved through continued research and development. As additive technology develops, many products (to save space and reduce transportation costs) will be created on demand (based on an appropriate digital model), similar to the way computing power is available on demand today. In this way, a kind of parallel digital world will form, «populated» by digital counterparts of inanimate objects and living beings. Interaction of these digital objects in virtual space, testing of the latter in various standard and abnormal situations will be aimed at prevention of possible accidents in the real world of real material objects.

\section{References}

1. Q. Qi, F. Tao, Y. Zuo, D. Zhao, Procedia CIRP, 72, 237-242, (2018)

2. D. Delen, H. Demirkan, Decis. Support. Syst. 55, 359-363 (2013), https://doi.org/10.1016/j.dss.2012.05.044

3. Z. Ben Miled, M.O. French, 55th AIAA Aerosp. Sci. Meet, American Institute of Aeronautics and Astronautics (2017), https://doi.org/10.2514/ 6.2017-0873

4. L. Hu, N. Ngoc-Tu, W. Tao, M.-C. Leu, X.F. Liu, M.R. Shahriar, S.M. Nahian Al Sunny, Proc. Manuf. 26, 1193-1203 (2018), https://doi.org/10.1016/j. promfg.2018.07.155

5. H. Millwater, J. Ocampo, N. Crosby, Eng. Fract. Mech. 221 (2019)

6. A.S. Orobinsky, A.A. Alukhanyan, R.A., Sychev, M.G. Kholina, European Research Studies Journal, 21, 333-349 (2018)

7. A. Kusiak, Nature, 544, 23-25 (2017)

8. J. Meierhofer, S. West, M. Rapaccini, C. Barbieri, Lect. Notes Bus. Inf. Process, 347359 (2020), https://doi.org/10.1007/978-3-030-38724-2_25

9. R. Bitton, T. Gluck, O. Stan, M. Inokuchi, Y. Ohta, Y. Yamada, T. Yagyu, Y. Elovici, A. Shabtai, Lect. Notes Comput. Sci. (Including Subser. Lect. Notes Artif. Intell. Lect. Notes Bioinformatics), 533-554 (2018)

10. E.H. Glaessgen, D.S. Stargel, Air force vehicles, Collect. Tech. Pap. AIAA/ASME/ASCE/AHS/ASC Struct. Struct. Dyn. Mater. Conf, 1-14 (2012) https://doi.org/10.2514/6.2012-1818

11. P.M. Karve, Y. Guo, B. Kapusuzoglu, S. Mahadevan, M.A. Haile, Eng. Fract. Mech. 225 (2020)

12. M. Macchi, I. Roda, E. Negri, L. Fumagalli, IFAC-PapersOnLine, 51, 790-795 (2018)

13. D. Mourtzis, J. Angelopoulos, N. Panopoulos, Procedia CIRP, 97, 331-336 (2021)

14. V.I. Gissin, M.A. Surzhikov, N.M. Serbulova, Lecture Notes in Networks and Systems, 198 (2021), https://doi.org/10.1007/978-3-030-69415-9_182 
15. [C.-A. Schumann, J. Baum, E. Forkel, F. Otto, 2017 Futur. Technol. Conf, 645-650 (2017)

16. N. Serbulova, et al, IOP Conf. Ser.: Earth Environ. Sci., 403 (2019), https://doi.org/10.1088/1755-1315/403/1/012127

17. C. Pylianidis, S. Osinga, I.N. Athanasiadis, Computers and Electronics in Agriculture, $184(2021)$

18. T. Deng, K. Zhang, Zuo-Jun (Max) Shen, JMSE, 6 (2021)

19. Z. Allam, D.S. Jones, Land Use Policy, 101 (2021)

20. V. Kurdyukov, S. Kanurny, E3S Web of Conf., 210 (2020), https://doi.org/10.1051/e3sconf/202021013005

21. N. Serbulova, et al, E3S Web Conf., 210 (2020), https://doi.org/10.1051/e3sconf/202021002005 